\title{
Comparative transcriptomics to understand light and temperature signalling across different plant species
}

\author{
Aditi Dwivedi*, KumudSaini and Aashish Ranjan \\ National Institute of Plant Genome Research, New Delhi
}

\begin{abstract}
Light and temperature signalling in Arabidopsis share strong parallels through the involvement of photoreceptors, phytohormones and transcription factors activity, suggesting common nodes between gene regulatory networks of these two signalling pathways. This is, further, supported by the phenocopy of thermomorphogenic responses to Shade Avoidance Responses (SAR) in Arabidopsis. We investigated the response of shade and high temperature in tomato (a dicot) and rice (a monocot). Similar to Arabidopsis, we observed shade induced elongation growth in both tomato and rice seedlings. However, high ambient temperature (HT) response differed across the three species. Contrary to hypocotyl elongation in Arabidopsis under HT, tomato seedlings did not show significant change in hypocotyl length while rice showed reduction in seedling length. We, then, investigated the underlying transcriptional responses of shade and high temperature across the three species using tissue-specific comparative transcriptome profiling by RNA sequencing. Differential expression analysis identified 1692 (shade) and 2664 (HT) differentially expressed genes (DEGs) in Arabidopsis, 2686 (shade) and 3614 (HT) DEGs in tomato while 2944 (shade) and 5549 (HT) DEGs in rice. We found that approximately 10-15\% DEGs were shared between shade and high temperature in these three species while $50-60 \%$ DEGs were unique to high temperature and $25-30 \%$ DEGs were unique to shade. Thus, despite sharing the phenotypic response and some common signalling pathways, the global transcriptional response of shade and high temperature in Arabidopsis are very unique. Further, high ambient temperature showed more pronounced impact on the transcriptional profile of all the three species compared to shade. Gene Ontology enrichment analysis revealed different hormones (auxin, gibberellin, brassinosteroid) biosynthesis and signalling, growth, development and cell cycle were major enriched categories in all the three species. Currently, we are establishing the conserved and diverged signalling pathways across the species employing orthologous gene relationship, and generating species-specific gene regulatory networks in response to shade and high temperature.
\end{abstract}

Keywords: SAR; thermomorphogenic; transcriptomic 